\title{
Reducing the need for carbon dioxide monitoring in the investigation of paediatric sleep disordered breathing
}

\author{
To the Editor:
}

There is increasing interest in the use of home sleep studies to diagnose paediatric sleep disordered breathing (SDB) as they are potentially more cost-effective, convenient and representative of the child's typical nights' sleep [1-3]. One concern is that most home sleep study equipment does not include measurement of carbon dioxide.

There are no current clear recommendations as to which subset of patients can be assessed using a home study and to date, no studies have specifically addressed the question of whether carbon dioxide monitoring needs to be included in paediatric SDB assessment. Home studies rely on the same cardiorespiratory polygraphy data as in-patient sleep studies (nasal cannulae and/or mouth thermistor for flow and snoring, thoracic and abdominal bands for respiratory effort, pulse oximeter and pulse rate sensor, and body position detector) but often without a carbon dioxide sensor. Therefore, we aimed to determine how often the addition of overnight carbon dioxide monitoring changed the clinical management of patients when added to cardiorespiratory polygraphy data. We hypothesised that $\mathrm{CO}_{2}$ data does not change management in otherwise healthy children who are being investigated for obstructive sleep apnoea (OSA) but is important in those with pre-existing medical conditions or comorbidities when added to cardiorespiratory polygraphy data.

We carried out a 2-year, retrospective analysis of children age $<18$ years who were referred to the Paediatric Sleep and Ventilation Unit at the Royal Brompton Hospital (London, UK) for investigation of SDB and who underwent a baseline cardiorespiratory polygraphy with measurement of transcutaneous carbon dioxide. We first made management recommendations blinded to the patient details and carbon dioxide data and then re-assessed after considering transcutaneous carbon dioxide. Age, sex, underlying disease and OSA symptoms were recorded. The same cardiorespiratory polygraphy equipment (SOMNOScreen Plus polygraphy set; SOMNOmedics, Randersacker, Germany) was used throughout the study period. Mean carbon dioxide was obtained through transcutaneous capnography (TCM CombiM monitor; Radiometer, Copenhagen, Denmark) and was considered abnormal if $>6.7 \mathrm{kPa}(>50 \mathrm{mmHg})$. Hypoventilation was defined as carbon dioxide $>6.7 \mathrm{kPa}(>50 \mathrm{mmHg}$ ) for $>25 \%$ of the recorded night as per American Association of Sleep Medicine rules [4]. As per European Respiratory Society guidelines, OSA was defined as an apnoea-hypopnoea index (AHI) $>1$ event per $\mathrm{h}$ in the presence of OSA symptoms and OSA severity was classified as mild, moderate or severe according to AHI [5]. The presence of symptoms of OSA such as snoring or witnessed apnoea was also recorded.

Patients' pre-existing medical conditions or comorbidities were classified as craniofacial abnormalities, chronic cough, laryngomalacia, asthma/wheeze, interstitial lung diseases, cystic fibrosis, congenital heart diseases, neurological/neuromuscular disorders and other syndromes, and obesity (table 1). Data were analysed using Prism version 7.02 (GraphPad, La Jolla, CA, USA). Descriptive statistics were generated on each measure. For all the reported variables, a test of normality was performed. For nonparametric data, median (interquartile range (IQR)) was reported. Multiple comparisons were performed via ANOVA or Kruskall-Wallis test according to data distribution.

There were 513 patients, 311 (61\%) of whom were male, with a median age of 4.5 years (IQR 2.37.9 years). 13 out of 513 were prescribed overnight oxygen; one was prescribed continuous positive airway

@ERSpublications

Overnight cardiorespiratory polygraphy without $\mathrm{CO}_{2}$ monitoring is adequate in children investigated for uncomplicated obstructive sleep apnoea http://ow.ly/P7Fs30lpKFj

Cite this article as: Trucco F, Bush A, Tan H-L. Reducing the need for carbon dioxide monitoring in the investigation of paediatric sleep disordered breathing. Eur Respir J 2018; 52: 1801290 [https://doi.org/ 10.1183/13993003.01290-2018]. 
TABLE 1 Clinical characteristics of study population

Subjects with OSA Median (IQR) $\mathrm{CO}_{2} \mathrm{kPa}$ symptoms
Subjects with changes after adding back $\mathrm{CO}_{2}$ data

$\begin{array}{cc}130 / 130 & 5.9(5.6-6.2) \\ 5 / 7 & 5.7(5.4-6.5) \\ 23 / 38 & 5.8(5.6-6.0) \\ 7 / 14 & 5.9(5.4-6.2) \\ 43 / 80 & 5.8(5.5-6.2) \\ 31 / 63 & 5.9(5.6-6.1) \\ 9 / 15 & 5.8(5.7-6.3) \\ 16 / 48 & 5.9(5.5-6.2) \\ 52 / 112 & 6.0(5.6-6.4) \\ 3 / 6 & 5.9(5.7-6.3) \\ 319 / 513 & \end{array}$

$0 / 130$

$1 / 7$

$0 / 52$

$1 / 14$

$1 / 80$

$1 / 63$

$0 / 15$

$2 / 48$

$12 / 112$

$2 / 6$

$20 / 513$
Outcome after adding back $\mathrm{CO}_{2}$ data

NA

CPAP $(1 / 1)$

NA

NIV (1/1)

NIV $(1 / 1)$

Further investigation (1/1)

NA

Start NIV (1/2)

Change $\mathrm{O}_{2}(1 / 2)$

Start NIV $(12 / 12)$

Start CPAP $(2 / 2)$

Patients are classified as "otherwise healthy" or "having pre-existing medical conditions or comorbidities". A breakdown of patients" pre-existing medical conditions or comorbidities, their overnight carbon dioxide values, cardiorespiratory polygraphy data and management post-sleep study are outlined. OSA: obstructive sleep apnoea; IQR: interquartile range; NA: not applicable; CPAP: continuous positive airway pressure; NIV: noninvasive ventilation.

pressure (CPAP) and oxygen; and one, noninvasive ventilation (NIV). 130 out of 513 were otherwise healthy children being investigated for OSA. 383 out of 513 had pre-existing medical conditions or comorbidities: craniofacial abnormalities, $\mathrm{n}=7$; chronic cough, $\mathrm{n}=38$; laryngomalacia, $\mathrm{n}=14$; asthma/ wheeze, $\mathrm{n}=80$; interstitial lung diseases, $\mathrm{n}=63$; cystic fibrosis, $\mathrm{n}=15$; congenital heart disease, $\mathrm{n}=48$; neurological/neuromuscular syndromes, $\mathrm{n}=112$; and obesity, $\mathrm{n}=6.189$ (49\%) out of 383 had clinical symptoms of OSA: five (71\%) out of seven with craniofacial abnormalities, 23 (61\%) out of 38 with chronic cough, seven (50\%) out of 14 with laryngomalacia, 43 (54\%) out of 80 with asthma/wheeze, 31 (49\%) out of 63 with interstitial lung diseases, nine (60\%) out of 15 with cystic fibrosis, 16 (33\%) out of 48 with congenital heart diseases, $52(46 \%)$ out of 112 with neurological/neuromuscular syndromes and three (50\%) out of six with obesity.

107 (82\%) out of 130 otherwise healthy patients were diagnosed with OSA from the baseline cardiorespiratory polygraphy results; 23 had a normal study. In these children without comorbidities with mild, moderate and severe OSA the prevalence of high mean carbon was none (0\%) out of 73, two (14\%) out of 14 and five (25\%) out of 20; the prevalence of hypoventilation was $13(18 \%)$ out of 73 , seven (50\%) out of 14 and $12(60 \%)$ out of 20; and the presence of rapid eye movement (REM)-related carbon dioxide elevation was 8 (11\%) out of 73 , seven (50\%) out of 14 and $13(65 \%)$ out of 20 , respectively. The addition of carbon dioxide data did not change assessment in any of these 107 children.

Conversely, in 20 (5\%) out of 383 children with pre-existing medical conditions or comorbidities, either abnormally high mean carbon dioxide levels (17 out of 20) or elevated carbon dioxide during REM sleep (three out of 20) changed management when these results were interpreted in conjunction with the cardiorespiratory polygraphy data. Median overnight carbon dioxide levels did not differ significantly across diagnostic groups $(\mathrm{p}=0.73)$ and did not significantly differ between children with or without pre-existing medical conditions or comorbidities $(\mathrm{p}=0.44)$.

In the group of children with pre-existing medical conditions or comorbidities, there were changes in recommendation after including carbon dioxide data to information from the cardiorespiratory polygraphy in one $(14 \%)$ out of seven patients with craniofacial abnormalities, none $(0 \%)$ out of 38 with chronic cough, one $(7 \%)$ out of 14 with laryngomalacia, one (1\%) out of 80 with asthma/wheeze, one (2\%) out of 63 with interstitial lung diseases, none $(0 \%)$ out of 15 with cystic fibrosis, two (4\%) out of 48 with heart disease, $12(11 \%)$ out of 112 with neurological/neuromuscular syndromes and two (33\%) out of six with obesity, respectively. Overall, in 18 (90\%) out of 20 children, CPAP or NIV was established. NIV was started in one out of one with laryngomalacia, one out of one with asthma/wheeze, one out of two patients with heart diseases and 12 out of 12 patients with neurological/neuromuscular syndromes; CPAP was established in one out of one patient with craniofacial abnormalities and two out of two patients with obesity. Finally, the addition of carbon dioxide to cardiorespiratory polygraphy prompted the safe up-titration of oxygen flow in one patient with heart disease and low baseline saturations. There were concerns that increasing oxygen therapy might result in an increase in his carbon dioxide levels; therefore, 
the study was performed on increased oxygen levels. Since carbon dioxide did not increase significantly, the child's home oxygen could be safely increased. In one patient with interstitial lung disease, unexpectedly high carbon dioxide levels lead to the instigation of further investigations.

In summary, in otherwise healthy patients suspected of having OSA, none had changes in clinical management with additional carbon dioxide data. Conversely, carbon dioxide abnormalities (either high mean carbon dioxide values or elevation during REM) can occur in patients with pre-existing medical conditions or comorbidities (20 (5\%) out of 383, particularly neurological/neuromuscular comorbidities, even without significant changes in the cardiorespiratory polygraphy. In 18 out of 20 patients, respiratory support was initiated as a result. A strength of this study is the large sample size $(>100)$. The main limitation of this study is the retrospective nature and we acknowledge that confirmatory prospective studies are needed. In conclusion, overnight carbon dioxide monitoring is not necessary for the diagnosis of SDB in otherwise healthy children but is crucial for the management of paediatric patients with pre-existing medical conditions or comorbidities. This is important because it means home cardiorespiratory polygraphy without transcutaneous carbon dioxide measurement can safely be used to diagnose OSA in otherwise healthy children, thus saving resources and reducing the need for admission.

Federica Trucco ${ }^{1,2}$, Andrew Bush ${ }^{1,3}$ and Hui-Leng $\operatorname{Tan}^{1}$

${ }^{1}$ Dept of Paediatric Respiratory Medicine, Royal Brompton Hospital, London, UK. ${ }^{2}$ DINOGMI, University of Genoa, Genoa, Italy. ${ }^{3}$ National Heart and Lung Institute, Imperial College, London, UK.

Correspondence: Federica Trucco, Dept of Pediatric Respiratory Medicine, Royal Brompton Hospital, Sydney St, SW3 6NP, London, UK. E-mail: federica_trucco@yahoo.it

Received: July 102018 | Accepted: Aug 062018

Conflict of interest: None declared.

\section{References}

1 Corral J, Sánchez-Quiroga MÁ, Carmona-Bernal C, et al. Conventional polysomnography is not necessary for the management of most patients with suspected obstructive sleep apnea. noninferiority, randomized controlled trial. Am J Respir Crit Care Med 2017; 196: 1181-1190.

2 Tan HL, Kheirandish-Gozal L, Gozal D. Pediatric home sleep apnea testing: slowly getting there! Chest 2015; 148: $1382-1395$.

3 Gozal D, Kheirandish-Gozal L, Kaditis AG. Home sleep testing for the diagnosis of pediatric obstructive sleep apnea: the times they are a changing. Curr Opin Pulm Med 2015; 21: 563-568.

4 Berry RB, Budhiraja R, Gottlieb DJ, et al. Rules for scoring respiratory events in sleep: update of the 2007 AASM Manual for the Scoring of Sleep and Associated Events. Deliberations of the Sleep Apnea Definitions Task Force of the American Academy of Sleep Medicine. J Clin Sleep Med 2012; 8: 597-619.

5 Kaditis AG, Alonso Alvarez ML, Boudewyns A, et al. Obstructive sleep disordered breathing in 2- to 18-year-old children: diagnosis and management. Eur Respir J 2016; 47: 69-94. 